\title{
Persistence in voting behavior: stronghold dynamics in elections.
}

\author{
Toni Pérez, Juan Fernández-Gracia, Jose J. Ramasco, and Víctor M. Eguíluz \\ Instituto de Física Interdisciplinar y Sistemas Complejos (IFISC) \\ http://www.ifisc.uib-csic.es \\ toni@ifisc.uib-csic.es
}

\begin{abstract}
Influence among individuals is at the core of collective social phenomena such as the dissemination of ideas, beliefs or behaviors, social learning and the diffusion of innovations. Different mechanisms have been proposed to implement inter-agent influence in social models from the voter model, to majority rules, to the Granoveter model. Here we advance in this direction by confronting the recently introduced Social Influence and Recurrent Mobility (SIRM) model, that reproduces generic features of vote-shares at different geographical levels, with data in the US presidential elections. Our approach incorporates spatial and population diversity as inputs for the opinion dynamics while individuals' mobility provides a proxy for social context, and peer imitation accounts for social influence. The model captures the observed stationary background fluctuations in the vote-shares across counties. We study the so-called political strongholds, i.e., locations where the votes-shares for a party are systematically higher than average. A quantitative definition of a stronghold by means of persistence in time of fluctuations in the voting spatial distribution is introduced, and results from the US Presidential Elections during the period 1980-2012 are analyzed within this framework. We compare electoral results with simulations obtained with the SIRM model finding a good agreement both in terms of the number and the location of strongholds. The strongholds duration is also systematically characterized in the SIRM model. The results compare well with the electoral results data revealing an exponential decay in the persistence of the strongholds with time.
\end{abstract}

\section{Introduction}

People making a decision in a ballot are expected to follow a rational behavior. Rational arguments based on utility functions (payoff) have been considered in the literature regarding vote modeling [1]2. The rational hypothesis, however, tends to consider the individuals as isolated entities. This might actually be the reason why it fails to account for relatively high turnout rates in elections [3]4]. The presence of a social context increases the incentive for a voter to actually vote, as he or she can influence several other individuals towards the same option 611]. This effect is not only restricted to turnout, but also applies to the 
choices expressed in the election [12. It is easy to find examples of people showing their electoral preferences in public in the hope of influencing their peers. Still social influence can also act in more subtle ways, without the explicit intention of the involved agents to influence each other. The collective dynamics of social groups notably differs from the one observed from simply aggregating independent individuals 13. Social influence is thus an important ingredient for modeling opinion dynamics, but it requires as well the inclusion of a social

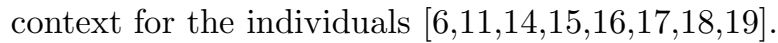

Even though nowadays the pervasive presence of new information technologies has the potential to change the relation between distance and social contacts, we assume that daily mobility still determines social exchanges to a large extent. Human mobility has been studied in recent years with relatively indirect techniques such as tracking bank notes [20] or with more direct methods such as tracking cell phone communications 21/22. A more classical source of information in this issue is the census. Among other data, respondents are requested by the census officers their place of residence and work. Census information is less detailed when considered at the individual level, but it has the advantage of covering a significant part of the population of full countries. Recent works analyzing mobile phone records have shown that people spend most of their time in a few locations 22. These locations are likely to be those registered in the census and, indeed, census-based information has been also used recently to forecast the propagation patterns of infectious diseases such as the latter influenza pandemic [23|24|25].

In this work, we follow a similar approach and use the recurrent mobility information collected in the US census as a proxy for individual social context. This localized environment for each individual accounts mostly for face-to-face interactions and leaves aside other factors, global in nature, such as information coming from online media, radio and TV. Our results show that a model implementing face-to-face contacts through recurrent mobility and influence as imperfect random imitation is able to reproduce geographical and temporal patterns for the fluctuations in electoral results at different scales.

\section{Definitions and Methods}

We have investigated the voting patterns in the US on the county level. We have used the votes for presidential elections in years 1980-2012. For each county $i=$ $1, \ldots, I$, we have data about the county geographic position, area, adjacency with other counties as well as data regarding population $N_{i y}$, and number of voters $V_{i y p}$ for each party $p$ for every election year $y=1, \ldots, Y$ (note that $\sum_{p} V_{i y p} \neq$ $N_{i y}$, since not everybody is entitled to vote). Raw vote counts are not very useful for comparing the counties as populations are distributed heterogeneously. Therefore we switch from vote counts to vote shares

$$
v_{i y p}=V_{i y p} / \sum_{p} V_{i y p}
$$


Since the votes received by parties others than Republicans and Democrats are minority, we have focused on the two main parties. We have further considered mostly relative voteshares $r_{i y p}$, which are absolute vote shares minus the national average for a given party every electoral year

$$
\begin{aligned}
r_{i y p} & =v_{i y p}-a_{y p}, \\
a_{y p} & =\sum_{i} v_{i y p} / I,
\end{aligned}
$$

where I stands for the number of counties.

The relative voteshares show how much above (positive) or below (negative) the national average are the results in given county.

Our main focus is on the persistence of voting patterns. We define a stronghold to be a county which relative voteshare remains systematically positive (or negative).

\subsection{Model definition and analytical description}

In the SIRM model $N$ agents live in a spatial system divided in non-overlapping cells. The $N$ agents are distributed among the different cells according to their residence cell. The number of residents in a particular cell $i$ will be called $N_{i}$. While many of these individuals may work at $i$, some others will work at different cells. This defines the fluxes $N_{i j}$ of residents of $i$ recurrently moving to $j$ for work. By consistency, $N_{i}=\sum_{j} N_{i j}$. The working population at cell $i$ is $N_{i}^{\prime}=\sum_{j} N_{j i}$ and the total population in the system (country) is $N=\sum_{i j} N_{i j}$. In this work, the spatial units correspond to the US counties and the population levels, $N_{i}$, and commuting flows, $N_{i j}$, are directly obtained from the 2000 census.

We describe agents' opinion by a binary variable with possible values +1 or -1 . The main variables are the number of individuals $V_{i j}$ holding opinion +1 , living in county $i$ and working at $j$. Correspondingly, $V_{i}=\sum_{l} V_{i l}$ stands for the number of voters living in $i$ holding opinion +1 and $V_{j}^{\prime}=\sum_{l} V_{l j}$ for the number of voters working at $j$ holding opinion +1 . We assume that each individual interacts with people living in her own location (family, friends, neighbors) with a probability $\alpha$, while with probability $1-\alpha$ she does so with individuals of her work place. Once an individual interacts with others, its opinion is updated following a noisy voter model 6/78910: an interaction partner is chosen and the original agent copies her opinion imperfectly (with a certain probability of making mistakes). A more detailed description of the SIRM model can be found in [26].

\section{Results}

Pursuing the topic of persistence and changes in opinion, as expressed by voting results, we have investigated the strongholds of both parties in USA. Figure 1 shows the spatial arrangement and the duration of the strongholds (measured in 
elections) for data of the US Presidential Elections during the period 1980-2012 and simulations using the electoral results of 1980 as initial condition. It can be seen at a glance, that the strongholds are not randomly distributed across the country, but clustered. This indicates that some form of correlation is present between the voting patterns. The republican strongholds seem to be concentrated mostly in the central-west, while democrat strongholds are dispersed mostly through the eastern parts, including urbanized areas. This is in agreement with the population distributions, republican strongholds being mostly lower populated counties, while democrats strongholds include some significant cities.

We quantify in Figure 2 the temporal evolution of the strongholds of the election data for the period 1980-2012 as well as for the strongholds forecasted by simulations after 9 elections. The influence of the commuting network and their interactions was revealed by contrasting the simulations results with and without network interaction. The evolution of the number of strongholds observed in the data at early stages is well described by the model with commuting interactions. For longer times, the model predicts that the number of strongholds will decay with time following an exponential law as shown in Figure 2. In the absence of commuting interaction, the number of strongholds decreases at a slower rate. Furthermore, the model overestimate the number of strongholds in this case since no other mechanisms than internal fluctuations act driving the county relative voteshare $r_{i y p}$ towards the average. Counties set as strongholds at the beginning of the simulation remains strongholds for a longer time.

We further test the accuracy of our model by computing the percentage of strongholds accurately predicted. As Figure 3 shows, the model with no commuting interactions reproduces a higher percentage of strongholds, however, it also gives higher and increasing number of false positives. On the contrary, the model with commuting interactions maintain a flat rate of false positive below $20 \%$. The accuracy of the model in this case remain higher than $50 \%$ after 7 elections.

Another question is what determines how long a county would be a stronghold for? We try to answer this question for the model by looking into the dependence of the strongholds duration with respect to the initial voteshare of the counties. As Figure 4 shows, there is a linear dependence for the duration of being a stronghold with the distance to the mean voteshare. Counties with initial larger deviation from the mean voteshare tend to be strongholds longer time. The fitting reveals that every tenth of relative voteshare corresponds on average to a stronghold duration of 5 elections.

\section{Discussion}

We have studied the persistence on the electoral system using the recently introduced Social Influence and Recurrent Mobility (SIRM) model for opinion dynamics which includes social influence with random fluctuations, mobility and population heterogeneities across the U.S. The model accurately predicts generic 


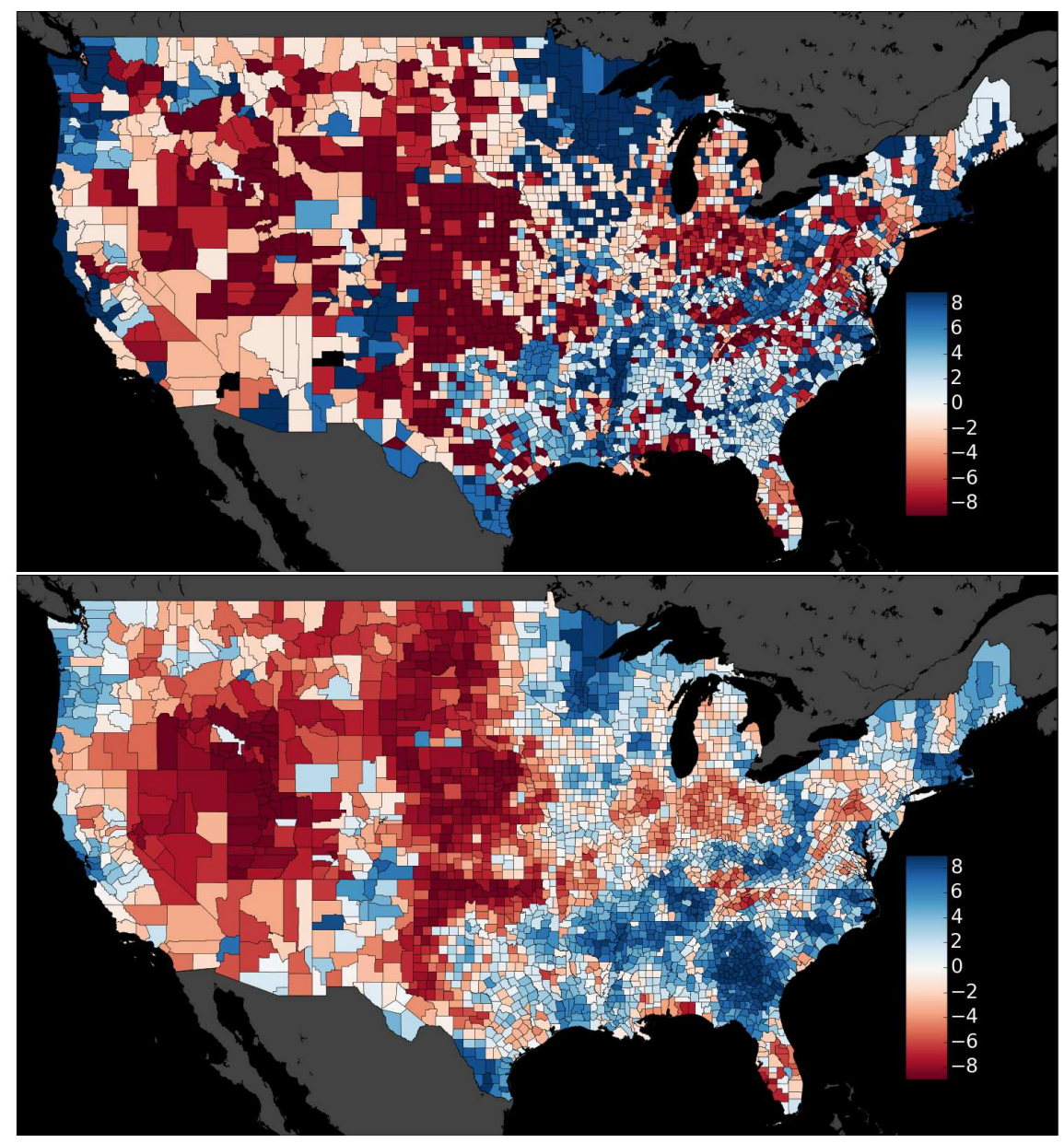

Fig. 1. Spatial distribution of the strongholds (red shows republicans while blue shows democrats). Codified by color the stronghold time measured in elections units (republicans stronghold times are given by the absolute value of the color bar). No county is a stronghold for both parties. Upper panel: US Presidential Elections data during the period 1980-2012. Bottom panel: model simulations for 9 elections using the electoral results from 1980 (at county level) as initial condition. 


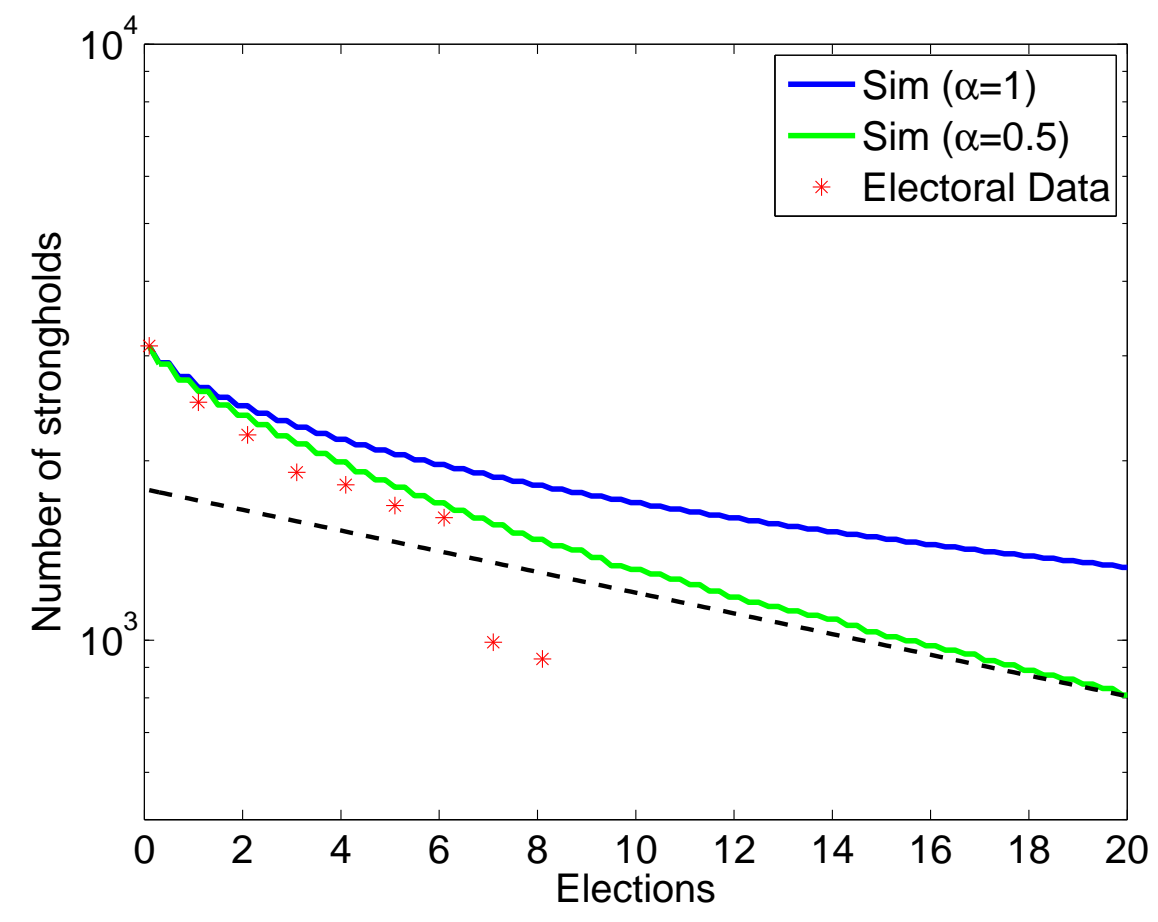

Fig. 2. Temporal evolution of the number of strongholds for electoral data and simulation results. Two different simulation results are presented, for $\alpha=0.5$ the individuals have the same probability of interacting with individuals from other county as with individuals of his own county, while for $\alpha=1$ interactions only within the same county would happen. Dashed line fits $f(t)=\frac{N_{c}}{2} \exp (-t / b)$ with $b=25$ elections. 


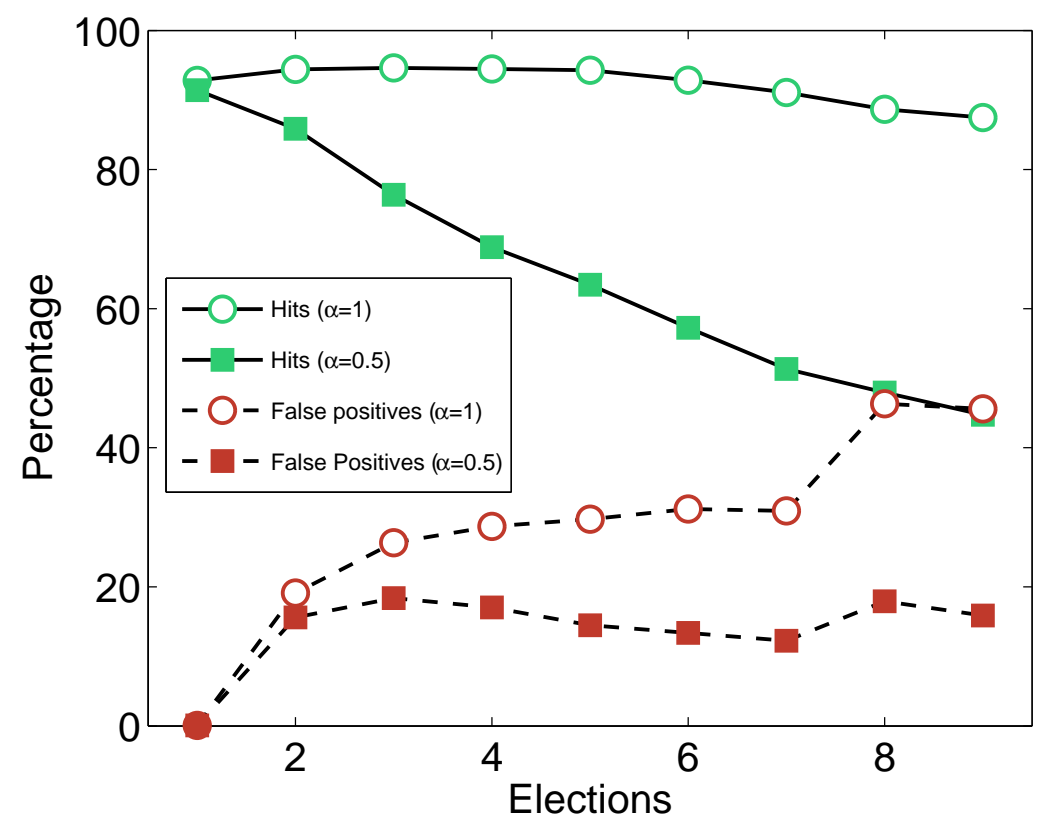

Fig. 3. Variation of the percentage of strongholds accurately predicted by the model (hits) for several elections. The number of false positives, i.e., counties identified as strongholds in the simulations without stronghold correspondence in the data is also shown. 


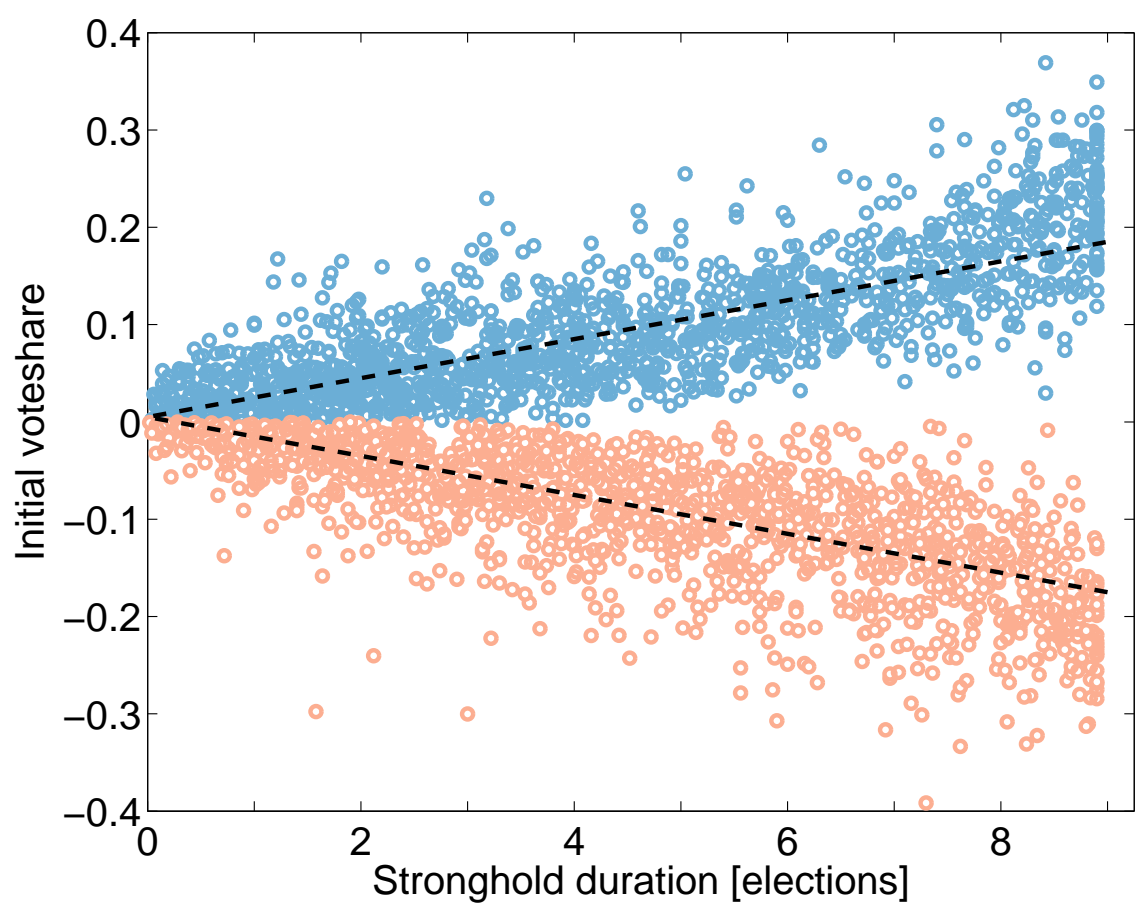

Fig. 4. Scatter plot of the initial voteshare versus the stronghold duration for model results. Red symbols shows republicans while blue symbols shows democrats. Dashed line correspond to the least square fitting revealing a symmetric linear behavior $y=$ $m x+n$ with $m= \pm 0.02$ and $n= \pm 0.005$. 
features of the background fluctuations of evolution of vote-share fluctuations at different geographical scales (from the county to the state level), but it does not aim at reproducing the evolution of the average vote-share.

We have contrasted the evolution of the number of strongholds of the election data for the period 1980-2012 with the strongholds forecasted by simulations finding a good agreement between them. The evolution of the number of strongholds observed in the electoral data at early stages is well described by the model with commuting interactions. However, the number of strongholds observed in the data changes abruptly after the presidential election of 2008. Our model is not able/does not intend to reproduce this behavior since it involves external driving forces not included in the model.

Our results also shows a good agreement between data and simulations for the location and duration of the strongholds. Strongholds are not randomly distributed across the country, but clustered. This indicates that some form of correlation is present between the voting patterns. The model reproduces nicely the spatial concentration of the both types of strongholds. Republican strongholds are mostly concentrated in the central-west, while democrat strongholds are dispersed mostly through the eastern parts, including urbanized areas.

As for the duration of the strongholds, we have found a linear dependence for the duration of being a stronghold with the distance to the mean voteshare. Counties with initial larger deviation from the mean voteshare tend to be strongholds for a longer time, on average, 5 elections for each tenth of relative voteshare. When commuting interactions are taken into consideration, our model exhibits an accuracy higher than $50 \%$ for up to 7 elections. The lack of this interactions causes an overestimation of the number of strongholds with an associated increase of the number of false positive decreasing the prediction accuracy of the model.

Our contribution sets the ground to include other important aspects of voting behavior and demand further investigation of the role played by heterogeneities in the micro-macro connection. Further elements will have to be included in order to produce predictions mimicking more accurately real electoral results. Some examples are the effects of social and communication media or the erosion of the governing party. The use of alternative communication channels is expected to affect voting behavior.

Acknowledgements. The authors acknowledge support from project MODASS (FIS2011-24785). TP acknowledges support from the program Juan de la Cierva of the Spanish Ministry of Economy and Competitiveness.

\section{References}

1. Riker W H, Ordeshook P C (1968) A theory of the calculus of voting Am Polit Sci Rev 62:25-42.

2. Gelman A et al (2012) Estimating the probability of events that have never occurred: When is your vote decisive? Journal of the American Statistical Association 93:1-9. 
3. B.C. Straits, Public Opin. Q. 54, 64-73 (1990).

4. C.B. Kenny, Am. J. Pol. Sci. 36, 259-267 (1992).

5. P.A. Allen, R.J. Dalton, S. Greene and R. Huckfeldt, Am. Polit. Sci. Rev. 96, 57-73 (2002).

6. J.H. Fowler, Turnout in a small world in Social Logic of Politics, ed. A. Zuckerman (Temple University Press), pp 269-287 (2005).

7. Holley R, Liggett T M (1975) Ergodic theorems for weakly interacting infinite systems and the voter model Annals of Probability 3:643-663.

8. Vazquez F, Eguíluz V M (2008) Analytical solution of the voter model on uncorrelated networks New J Phys 10:063011.

9. Sood, V. and Redner, S. (2005) Voter model on heterogeneous graphs Phys. Rev. Lett. 94:178701.

10. P.L. Krapivsky, S. Redner and E. Ben-Naim A Kinetic View of Statistical Physics (Cambridge University Press) (2010)

11. R.M. Bond, C.J. Fariss, J.J. Jones, A.D.I. Kramer, C. Marlow, J.E. Settle and J.H. Fowler, Nature 489, 295-298 (2012).

12. A.S. Zuckerman The Social Logic Of Politics: Personal Networks As Contexts For Political Behavior (Temple University Press) (2005).

13. J. Lorenz, H. Rauhut, F. Schweitzer and D. Helbing, Proc. Natl. Acad. Sci. USA 108, 9020-9025 (2011).

14. E.M. Rogers, Diffusion of Innovations (Free Press, New York) (1995).

15. C. Castellano, S. Fortunato and V. Loreto, Rev. Mod. Phys. 81, 591-646 (2009).

16. N.A. Christakis and J.H. Fowler, N. Engl. J. Med. 357, 370-379 (2007).

17. A.L. Hill, D.G. Rand, M.A. Nowak and N.A. Christakis, Proc. Biol. Sci. 277, 3827-3835 (2010).

18. L. Rendell, R. Boyd, D. Cownden, M. Enquist, K. Eriksson, M.W. Feldman, L. Fogarty, S. Ghirlanda, T. Lillicrap and K.N. Laland, Science 328, 208-213 (2010).

19. D. Centola, Science 329, 1194-1197 (2010).

20. Brockmann D, Hufnagel L, Geisel T (2006) The scaling laws of human travel Nature 439:462-465.

21. M.C. González, C. Hidalgo and A.-L. Barabási, Nature 453, 779-782 (2008).

22. C. Song, Z. Qu, N. Blumm and A.-L. Barabási, Science 327, 1018-1021 (2010).

23. D. Balcan, V. Colizza, B. Gonçalves, H. Hu, J.J. Ramasco and A. Vespignani, Proc. Natl. Acad. Sci. USA, 106, 21484-21489 (2009).

24. M. Tizzoni, P. Bajardi, C. Poletto, J.J. Ramasco, D. Balcan, B. Gonçalves, N. Perra, V. Colizza and A. Vespignani, BMC Medicine 10, 165 (2012).

25. Sattenspiel L, Dietz K (1995) A structured epidemic model incorporating geographic mobility among regions Math Biosci 128:71-91.

26. Juan Fernández-Gracia, Krzysztof Suchecki, José J. Ramasco, Maxi San Miguel, and Víctor M. Eguíluz, Phys. Rev. Lett. 112, 158701 (2014). 